\title{
Pest Control in Primary Sector: Towards the Identification of Knowledge Gaps
}

\author{
Angeliki Stefopoulou ${ }^{1}{ }^{\circledR}$, Dionyssia A. Maselou ${ }^{1}$, Dimitrios Papachristos ${ }^{1}{ }^{\circledR}$, Antonios Kolimenakis ${ }^{1}{ }^{\circledR}$, \\ Antonios Michaelakis ${ }^{1}$ (D), Christos Athanassiou ${ }^{2}$ and George Vlontzos ${ }^{2, *(D)}$ \\ 1 Department of Entomology and Agricultural Zoology, Benaki Phyropathological Institute, \\ 14561 Kifissia, Greece; a.stefopoulou@bpi.gr (A.S.); d.maselou@aua.gr (D.A.M.); d.papachristos@bpi.gr (D.P.); \\ akolimenakis@gmail.com (A.K.); a.michaelakis@bpi.gr (A.M.) \\ 2 Department of Agriculture, Crop Production and Rural Development, School of Agricultural Sciences, \\ University of Thessaly, 38446 Volos, Greece; athanassiou@uth.gr \\ * Correspondence: gvlontzos@agr.uth.gr
}

Citation: Stefopoulou, A.; Maselou, D.A.; Papachristos, D.; Kolimenakis, A.; Michaelakis, A.; Athanassiou, C.; Vlontzos, G. Pest Control in Primary Sector: Towards the Identification of Knowledge Gaps. Agronomy 2021, 11, 1596. https://doi.org/10.3390/ agronomy11081596

Academic Editor: Luca Ruiu

Received: 1 June 2021

Accepted: 9 August 2021

Published: 11 August 2021

Publisher's Note: MDPI stays neutral with regard to jurisdictional claims in published maps and institutional affiliations.

Copyright: (c) 2021 by the authors. Licensee MDPI, Basel, Switzerland. This article is an open access article distributed under the terms and conditions of the Creative Commons Attribution (CC BY) license (https:// creativecommons.org/licenses/by/ $4.0 /)$.

\begin{abstract}
Pest control provides important and useful services in rural and urban environments, activities, and value chains. Despite the significance of them, under operational, environmental and food security and safety terms, there are still gaps in the awareness level of targeted groups. Therefore, in the current work, we have carried out questionnaire-based surveillance to pest control operators in Greece and Cyprus, in an attempt to illustrate their knowledge regarding pest control in conjunction with demographic characteristics. In this context, the results underlined the need for further training in all aspects of pest control, but also relative activities that are often are operated by pest controllers, such as food safety activities and applications in the urban and sub-urban environment. The replies that had been obtained during the survey showed good correlations of knowledge with specific variables, such as the size of the company, the age group, and the educational background, in conjunction with the country in which the professional activities are carried out. To our knowledge, this is the first work of its kind, in terms of pest controllers' perceptions and selfevaluation, illustrating specific knowledge gaps that have to be alleviated towards improved services in urban pest control, especially in sensitive urban areas and facilities, and improved measures for safety during the applications.
\end{abstract}

Keywords: pest control operators; food safety; urban pests; public health; safety; biocides; agriculture; factor analysis

\section{Introduction}

Questionnaire-based surveys consist of useful tools to record the effects of different systems and process characteristics and to evaluate the dynamic changes towards this direction at both professional/industrial and citizen scale, [1]. Despite the fact that pest control is a key element in food safety, worker safety, and various aspects of industrial operations and activities, it is often neglected as a necessity in the urban and suburban environment. Indicatively, pest controllers applications focus on pests and diseases in houses and also in different social structures (schools, hotels, hospitals, etc.) and as such, their applications are directly related to factors that may endanger public/human health (ref is needed). The term "pest controllers" implies the group of professionals that deal with biocide use, i.e., the use of specialized formulations that are registered for use exclusively to the urban and sub-urban environment, or in specific scenarios of area-wide management. Thus, biocide use is a very specialized procedure, as opposed to the use of plant protection products that are applied much wider, in most cases by non-specialized operators, i.e., farmers [2]. Indicatively, the applications for the control of mosquitoes- that are vectors of important diseases- in the sub-urban environment is usually operated by pest controllers, as it requires specific biocides and targeted-applications [3]. In this regard, pest control 
requires a multiscale operational plan that includes all the different contexts that require the involvement of professional pest controllers.

In terms of pest controllers' training requirements and knowledge gaps, there are serious diverging approaches, arising from different legislation and regulatory aspects per country. Hence, some countries require a university/technical degree for licensed pest control operators, while others require the successful completion of a series of training courses but no degree [4]. Also, the problems are different per area, e.g., there are areas where "urban pests", such as cockroaches and rodents are the major pest problems and areas where insect vectors, such as fleas and mosquitoes cause serious problems [5]. This fact also differentiates the types of pest controllers and categorizes them according to their specialization; still, in many countries, the authorities provide only one type of license, regardless of the target structures and the application scenarios. This consists of an additional implication in training, as each category has specific requirements that need to be covered. Knowledge gaps in pest control is a fact, often underestimated along with other similar operations, such as food safety [6,7]. Behavioral studies are a widely recognized and reliable methodological tool for identifying groups of different incentives leading to adequate decisions or actions [8]. The diversity of pests affecting urban and rural activities, combined with the dynamic situation of active ingredients (chemical or not) applied through disinfestation protocols, formulate a quite challenging context for pest controllers to adapt and provide high-quality services. The complexity of the sector, combined with the significant challenges the relative value chains are facing, require innovative assessment methods for identifying the operational status of pest controllers, as well as the efficacy of ongoing training sessions, curricula, or educational material. This innovation is based on the bottom-up approach of this survey, by asking the professionals of the sector to assess training courses, applying as main criterion their successful response to the market requirements. This approach quantifies the 'distance' between the perceptions of the trainers about the needs of the market and the sector, and the actual requirements derived from them.

Therefore, the objective of the current study is to evaluate knowledge gaps of a wide group of pest controllers that are professionally active in Greece and Cyprus, based on a questionnaire-survey that elucidated their beliefs, in relation to key demographic characteristics and indicators, in an attempt to share these gaps for a targeted training strategy. The significance of analyzing this topic is mainly related to the level of understanding the unique nature of pest control, different from pesticidal applications that are carried out in crops/orchards.

\section{Materials and Methods}

\section{Factor Analysis}

Principal Component Analysis (PCA) was used to further process the data, which is a method by which individuals' tendencies, perceptions, and beliefs on specific issues are examined [9-12]. Although this method reduces the information available in order to group it, it manages to produce a valid and understandable result.

In order to carry out the PCA some conditions must first apply:

- Variables are quantitative or expressed on a scale, such as whether a person is happy with a product, on a scale of -2 to 2 , with -2 being a completely negative experience while 2 being a completely enjoyable experience.

- The number of answers to be analyzed is also significant. In no case should it be below 50 and it should be well above 100. In addition, it is desirable that the number of responses is not less than 10 per variable while the preferred practice is to be more than 20. In this sample, we have 171 observations for 22 factors, so it is $171 / 22=7.78$. However, the fact that the sample consistency index is extremely high $(\mathrm{KMO}=0.923)$ enables the processing of database data by this method.

- Observations should be independent [13].

The above conditions are being assessed by the application of the following indicators: 
- The Kaiser-Meyer-Olkin Index (KMO) [14,15].

- Barlett's Sphericity test [10].

The Kaiser-Meyer-Olkin Index (KMO) assesses the suitability of the sample to be analyzed. In particular, it examines whether the relative size of the correlation coefficients is related to the partial correlation coefficients. Generally, the greater this correlation, the better the sample to be analyzed. When the value of KMO is too low, less than 0.5 means that factor analysis will not yield satisfactory results. Values above 0.7 are considered relatively good for analysis while 0.8 are excellent for analysis. To explain the PCA Scoreboard, it is important to mention two concepts: the factor loading and the percentage of variation of the main components. The participation index interprets the extent to which a dataset affects the formatted factor. If the turnout ratio is low enough, below 0.4 , then the influence it has is considered to be very small and therefore rejected, from 0.5 to 0.7 , the turnout rate is considered satisfactory, while from 0.7 and above, it may be explaining a great deal of the factors. On the other hand, the equity index explains whether this factor is involved in solving the given problem. It is worth emphasizing that in this research, due to the limit of responses per variable, all factor loadings are $>0.6$, indicating the validity of the results.

With the Barlett sphericity test, it is examined whether the observed correlation of the dataset, even if $R=(r i j)(p \times p)$, is statistically significantly different from its real identity. Therefore, the null hypothesis $(\mathrm{H} 0)$ is that the data is arranged in a rectangular form. This assumption is checked at a $5 \%$ significance level [16].

As can be seen from Table 1 below, the sample is excellent for analysis by the PCA method, as the KMO is excellent at 0.923 and the Barlett sphericity test is also highly significant $(=0.000)$.

Table 1. Barlett KMO and sphericity test results.

\begin{tabular}{ccc}
\hline & KMO and Bartlett'sTest \\
\multicolumn{2}{c}{ Kaiser-Meyer-Olkin Measure of Sampling Adequacy } & $\mathbf{0 . 9 2 3}$ \\
\hline Bartlett'sTest of Sphericity & Approx. Chi-Square & 3274.040 \\
& Df & 231 \\
& Sig. & 0.000 \\
\hline
\end{tabular}

3. Results

Quantitative Characteristics of the Sample

The questionnaires were distributed to pest professionals, professionals in the field of biocides as well as other professionals involved with pest control, between June and July 2016. There were 419 recipients (326 recipients were professionals in pest management, 47 recipients from the field of biocides, 14 recipients from research institutes occupied with pest control research, and 32 recipients from the ministry of Agriculture). A total of 168 responses were received.

The demographic characteristics of the sample indicate that the majority of the sample were young people, as $84.5 \%(n=168)$ were under 50 years of age (Figure 1). In regard to education levels, the majority had a high level of education, with the highest proportion to hold a higher education diploma, and a high percentage already having a postgraduate or doctoral degree (Figure 2).

In addition, $80 \%$ of the interviewees said that they had already participated in training courses in pest management, while the percentage of those who had no prior training in the subject was very low. It is noteworthy to mention that most of the respondents answered that they had internal incentives to attend this training, with $66 \%$ of them deciding on an individual basis to participate, and 33\% of them taking part in the initiative of the business they work for. Therefore, these are the major groups of incentives, motivating them to join training sessions on pest control management. The following Table 2, illustrates the results obtained from the PCA application. 


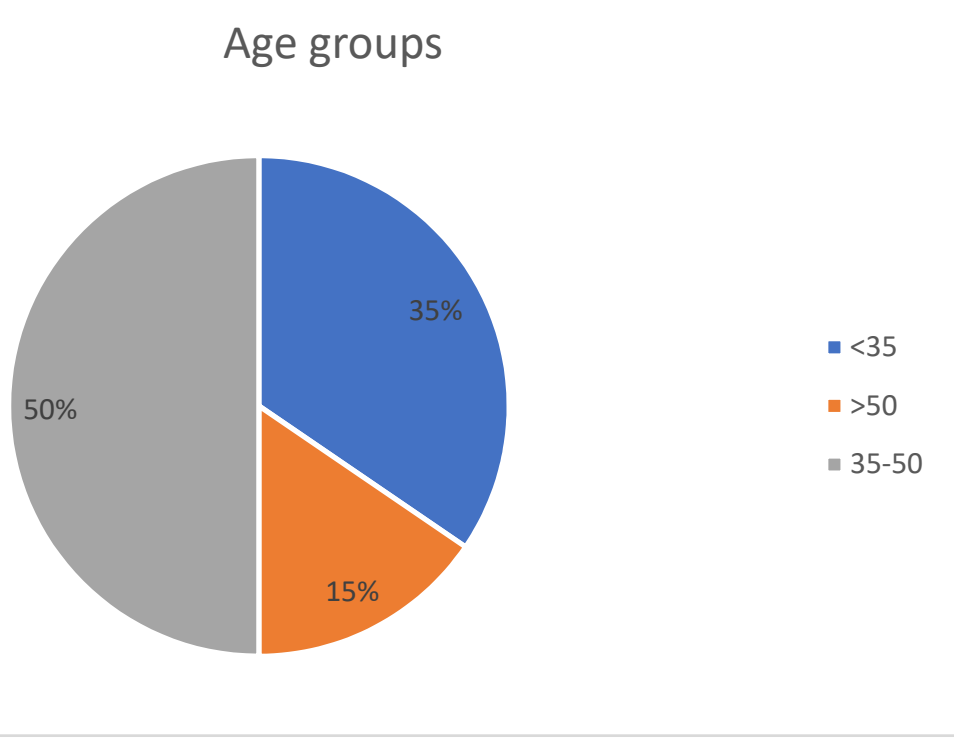

Figure 1. Illustration of sample age groups.

\section{Educational level}

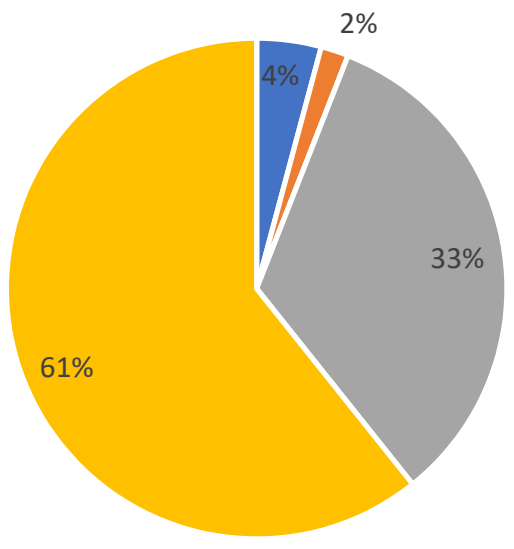

- Hihg school graduates

- College graduates

- Postgraduate graduates

- BSc graduates

Figure 2. Illustration of educational level of the sample.

PCA analysis resulted in two district factors, explaining on a satisfactory level the decision-making process of the interviewees. More specifically:

Factor 1: Knowledge is shown to be the first important factor. It appears that people involved in this survey believe that knowledge of issues such as protective means, active ingredients, legislation, environmental impacts, and the need to learn new methods and techniques is extremely necessary. The first Factor consists of 15 questions in total, highlighting its importance. "Factor 1"'s contribution is quite high, at $43.4 \%$.

Factor 2: Safety is the second most important factor. Security, in terms of people working in the disinfestation area, as well as active ingredients, use protocols and secure application protocols are the main issues of concern for the professionals of this sector. Factor 2 consists of 7 questions, a number that is sufficient to be considered valid. "Factor 2 "'s contribution to the overall problem approach is $21.5 \%$, explaining, in combination with Factor 1, 64.9\% of the problem approach. Surprisingly, question Q7 (Any company active in the field of insecticides should have responsibility for health and safety issues) has the smallest contribution to the Safety Factor. 
Table 2. Results of Factor Analysis (PCA).

\begin{tabular}{|c|c|c|}
\hline $\begin{array}{l}\text { Q16. Knowledge of precautionary measures in the application of biocide } \\
\text { products in order to effectively protect the practitioner non-users and } \\
\text { the environment }\end{array}$ & 0.800 & \\
\hline $\begin{array}{l}\text { Q14. Knowledge of the properties of active ingredients of biocides and their } \\
\text { possible effects on public health and the environment }\end{array}$ & 0.771 & \\
\hline $\begin{array}{l}\text { Q12. Knowledge on the various methods of control of pests and products } \\
\text { available on the market }\end{array}$ & 0.769 & \\
\hline $\begin{array}{l}\text { Q15. Knowledge of the legislation on the proper and safe use of biocide } \\
\text { products (active ingredients and preparations) and for the application } \\
\text { of insecticides }\end{array}$ & 0.768 & \\
\hline Q22. Field training (practical application of methods) & 0.767 & \\
\hline $\begin{array}{l}\text { Q10. Knowledge of the best management-response techniques applied to the } \\
\text { types of target organisms. }\end{array}$ & 0.763 & \\
\hline Q21. Mixed training (Including meetings and e-learning) & 0.753 & \\
\hline Q19. Attendance of scientific workshops/conferences & 0.732 & \\
\hline Q8. Appropriate identification of targeted pests. & 0.730 & \\
\hline $\begin{array}{l}\text { Q13. Understanding the biocide label markings (category, toxicity, } \\
\text { safety, etc.) }\end{array}$ & 0.728 & \\
\hline $\begin{array}{l}\text { Q17. Knowledge of safety measures/methods for storage, transport and } \\
\text { handling of empty packaging (biocide products) }\end{array}$ & 0.727 & \\
\hline Q9. Knowledge of the biology and ecology of pests. & 0.702 & \\
\hline $\begin{array}{l}\text { Q11. Knowledge of non-chemical methods that can be adopted as } \\
\text { target-pest management measures }\end{array}$ & 0.668 & \\
\hline Q20. Online education (e-learning) & 0.648 & \\
\hline Q18. Classroom education (Trainer training) & 0.641 & \\
\hline $\begin{array}{l}\text { Q2. There is a need for safety instructions for those operating in the } \\
\text { disinfestation area }\end{array}$ & & 0.813 \\
\hline $\begin{array}{l}\text { Q5. Staff training/education on the safe use of biocides for public health and } \\
\text { the environment is required. }\end{array}$ & & 0.799 \\
\hline Q4. Staff information/training on occupational safety issues is required. & & 0.789 \\
\hline Q3. Information/training of staff on new services/methods is required, & & 0.771 \\
\hline $\begin{array}{l}\text { Q1. Occupational safety during disinfestation plays a key role for an } \\
\text { insecticide company }\end{array}$ & & 0.758 \\
\hline $\begin{array}{l}\text { Q6. Co-operation with external consultants/trainers (specialists in the field) } \\
\text { is required. }\end{array}$ & & 0.706 \\
\hline $\begin{array}{l}\text { Q7. Every company operating in the field of insecticides should be } \\
\text { responsible for health and safety issues. }\end{array}$ & & 0.600 \\
\hline Percentage of Main Components Fluctuation & 43.4 & 21.5 \\
\hline
\end{tabular}

In addition, the two factors were examined in relation to the socio-economic characteristics of the sample. Age, education, size of business, and work experience consist the parameters of it. In Table 3 it was found that both factors were not correlated with most of these variables. The exception is the correlation of Security with the size of business, with a very strong correlation and exceptional levels of importance. 
Table 3. Aggregate Table of Correlations between Factors and Sample Variables.

\begin{tabular}{ccc}
\hline & Knowledge & Security \\
\hline Age & - & - \\
\hline Educational level & - & - \\
\hline Business Size & - & $* * *$ \\
\hline Work experience & - & - \\
\hline
\end{tabular}

*** Statistical significance at 0.01 level.

Additionally, the ANOVA Table 4 reveals that the model fits extremely well, with a significance of 0.000 , while Table 5 shows the very strong correlation of the size of the firm with security issues. Both Tables 4 and 5 verify the parameters enhancing the effectiveness of training, as well as the priorities providing stronger incentives for participation.

Table 4. Coefficients.

\begin{tabular}{cccccc}
\hline \multicolumn{7}{c}{ ANOVA } \\
\hline \multicolumn{2}{c}{ Sum of Squares } & df & Mean Square & F & Sig. \\
\hline Regression & 25.517 & 2 & 12.758 & 21.802 & $0.000^{\mathrm{b}}$ \\
Residual & 96.555 & 165 & 0.585 & & \\
Total & 122.071 & 167 & & & \\
\hline
\end{tabular}

b Statistical significance at 0.01 level.

Table 5. ANOVA results with dependent variable the size of the firm.

\begin{tabular}{cccccc}
\hline \multicolumn{2}{c}{ Unstandardized Coefficients } & \multicolumn{2}{c}{ Unstandardized Coefficients } \\
\hline & B & Std. Error & Beta & t & Sig \\
\hline (Constant) & 1.607 & 0.059 & & 27.231 & 0.000 \\
\hline Health & -0.002 & 0.059 & -0.003 & -0.041 & 0.967 \\
\hline Safety & -0.391 & 0.059 & -0.457 & -6.603 & 0.000 \\
\hline
\end{tabular}

\section{Discussion}

According to the study's results, the two major factors determining pest controllers' attitude towards training are Knowledge and Safety. In this context, the respondents identified specific knowledge gaps directly associated with their training, mostly emphasizing the necessity for applying field training and mixed training methods. This indicates the need for considerable modifications and additional requirements for the majority of the already existing training courses. Usually, the rationale of them is based on theoretical approaches. In this context, additional work is needed to set the baseline for an innovative training system, tailored to the needs of the market and the professionals, which should satisfy target audiences with different training and knowledge requirements. In addition, due to the wide range of pest control activities, they should also cover "classical" pest control needs (e.g., cockroaches in the urban environment, etc.), combined with other topics related to food safety (and the industrial-scale) and mosquito control (in an area-wide management approach). This is feasible if different modules target different groups of pest controllers, providing them the ability to 'build' their training portfolio according to their specific needs. In this regard, training packages should cover the required needs and ecosystems, i.e., urban, sub-urban, area-wide, and application scenarios. The current findings are in accordance with the recent results reported by Djekic et al. [6], illustrating knowledge gaps in food safety and pest control in companies from Greece and Serbia.

Table 4 suggests the different approaches of the respondents regarding the Security issue. The size of firms is fully correlated with safety issues, proving that there are distinct organizational differences among firms, having probably as an origin the complexity of 
the contracts each firm has to execute and the services to deliver. This is particularly important, as pest controllers often handle compounds and formulations that have high mammalian toxicity and may pose certain environmental risks, which requires additional attention in training modules and attributes. In a recent survey at the European level Djekic et al. (2019b) [7], underlined the importance of the size of the firm in terms of following best management practices, in regard to awareness about food safety, with larger companies clearly showing increased awareness towards this direction in comparison with smaller ones.

\section{Conclusions}

It is worth mentioning that the results of this study are based on the beliefs of those involved in the disinfestation industry. In other words, the evaluation was not based on consumers of the services in question, or by a third-party public or private entity, which may have had limited knowledge or understanding of the particularities of the sector but elicited directly through the operators of the sector [17-19]. These attributes seem to be related to the profile of the different pest controllers (country of origin, educational background, etc.), but there are some generalizations that can be drawn, such as their mutual recognition of knowledge gaps. The results of the present work can be further improved on the basis of a strategy that fully responds to industrial needs and can be applied with success in various target audiences of pest controllers.

In view of the above, a continuous need emerges for information and training on practices that will be useful in the professional development of those involved in this service sector. This can be achieved by establishing partnerships with accredited bodies such as universities and institutes that already possess the know-how, but also have the necessary infrastructure to share this knowledge in a simple and comprehensive way. Through this process trainees will gain all the necessary knowledge, both theoretically and in practice, from an official and recognized body. Additionally, the institutions through this process will come closer to the modern needs and concerns of the field, continually improving the quality of learning they provide. With the demonstration of these disinfestation protocols, they will satisfy their need for real knowledge. The knowledge they gain could also provide them with greater levels of safety in their workplace.

Surprisingly, from the range of factors that have been examined, as noted above, the size of firms was not positively correlated with any variable, with the exception of safety. Hence, while respondents directly assess low importance (0.600), as a result of factor analysis, when applying a more sophisticated statistical analysis (ANOVA + Factor Analysis), the information shared by respondents indicates the need to truly enhance the safety of application and use of insecticides and related biocide formulations. Such a finding could be characterized as a positive and promising one, since the existence of more complex operating structures of a business is on the right path, leading to a proportionate increase in its size. In a large business, it is possible to define the responsibilities that each person assumes, which is not easy and practically feasible in small businesses. In large companies, it is possible for their executives to be highly specialized in specific activities and can focus their activities on a discrete role that they will be able to perform with maximum success rates. It is obvious that this is not feasible in small businesses because of the large volume of responsibilities it has for its executive capacity. It also makes it clear that a large business is able to provide the necessary equipment to its members based on the protocols for the proper use of chemicals ingredients, has the ability to control and modernize this equipment whenever necessary, while a small business may not be able to meet these requirements.

In light of our findings, it becomes evident that there is an urgent need to initiate a dialog among these companies but also among the industry, the legislators/regulators, and the stakeholders, on the establishment and operation of larger schemes through a beneficial process for all parties, with a view to develop increased competitiveness, but above all providing the necessary security measures to members, but also to their customers. It 
is of the utmost importance that there is at least one person responsible for health and safety issues because this is the only way to achieve real and highly guaranteed safety. Moreover, given the nature of pest control that deals with extremely sensitive environments (i.e., hospitals, schools, etc.), it is essential to establish specific safety procedures that will include actions that have to be followed after the application, such as recycling of the empty biocide containers, deactivation of rodenticides, etc.

Author Contributions: Conceptualization, A.M., D.P. and A.S.; formal analysis, G.V. and A.S.; funding acquisition, A.M.; investigation, A.M., C.A., A.S. and D.A.M.; methodology, A.M., C.A., A.S., A.K., G.V. and D.A.M.; project administration, A.M.; resources, A.M., C.A. and G.V.; supervision, A.M., D.P., C.A. and G.V.; validation, A.M., C.A., D.P. and G.V.; writing—original draft, G.V.; writingreview and editing, A.M., C.A., A.K. and G.V. All authors have read and agreed to the published version of the manuscript.

Funding: This research has been co-financed by the European Union (Leonardo da Vinci- Transfer of Innovation) and Greek national funds through the Pest Practice project "Advancing work safety for pest control workers" (2013-1-GR1-LEO 05-13904). The funders had no role in study design, data collection and analysis, decision to publish, or preparation of the manuscript.

Institutional Review Board Statement: Not applicable.

Informed Consent Statement: Not applicable.

Data Availability Statement: Not applicable.

Conflicts of Interest: The authors declare no conflict of interest.

\section{References}

1. Kleisiari, C.; Duquenne, M.-N.; Vlontzos, G. E-Commerce in the Retail Chain Store Market: An Alternative or a Main Trend? Sustainability 2021, 13, 4392. [CrossRef]

2. Wittmer, I.K.; Bader, H.-P.; Scheidegger, R.; Singer, H.; Lück, A.; Hanke, I.; Carlsson, C.; Stamm, C. Significance of urban and agricultural land use for biocide and pesticide dynamics in surface waters. Water Res. 2010, 44, 2850-2862. [CrossRef] [PubMed]

3. Brühl, C.A.; Després, L.; Frör, O.; Patil, C.D.; Poulin, B.; Tetreau, G.; Allgeier, S. Environmental and socioeconomic effects of mosquito control in Europe using the biocide Bacillus thuringiensis subsp. israelensis (Bti). Sci. Total Environ. 2020, 7, 24. [CrossRef] [PubMed]

4. Schidgmall, R.S. Club controllers requirements: Skills, knowledge, and responsibilities. Bottomline 1998, 12, 14-18.

5. Sapunov, V.B. Clean Ecological Methods of Struggle with Urban Pests. Am. J. Mod. Phys. 2021, 10, 26-29. [CrossRef]

6. Djekic, I.; Kavallieratos, N.G.; Athanassiou, C.G.; Jankovic, D.; Nika, E.P.; Rajkovic, A. Pest control in Serbian and Greek food establishments: Opinions and knowledge. Food Control. 2019, 98, 281-289. [CrossRef]

7. Djekic, I.; Mujcinovic, A.; Nikolic, A.; Jambrak, A.R.; Papademas, P.; Feyissa, A.H.; Kansou, K.; Thomopoulos, R.; Breisen, H.; Kavallieratos, N.G.; et al. Cross-European analysis on the use of mathematical models in food industry. J. Food Eng. 2019, 261, 109-116. [CrossRef]

8. Burton, R.J. Reconceptualising the 'behavioural approach'in agricultural studies: A socio-psychological perspective. J. Rural Stud. 2004, 20, 359-371. [CrossRef]

9. Syms, C. Principal Components Analysis. In Encyclopedia of Ecology; Elsevier: Amsterdam, The Netherlands, 2018.

10. Sainani, K.L. Introduction to Principal Components Analysis. PMER 2014, 6, 275-278.

11. Chege Gabriel, K. Oblique versus Orthogonal Rotation in Exploratory Factor Analysis. Int. J. Res. Sci. Innov. 2019 , 6, $212-216$.

12. Hair, J.F.; Black, W.C.; Babin, B.J.; Anderson, R.E. Multivariate Data Analysis; Prentice-Hall: Hoboken, NJ, USA, 2010; ISBN 978-0138132637.

13. Bro, R.; Smilde, A.K. Principal Component Analysis. Anal. Methods 2014, 6, 2812-2831. [CrossRef]

14. Nkansah, B.K. On the Kaiser-Meier-Olkin's Measure of Sampling Adequacy. Math. Theory Model. 2011, 8, 52-76.

15. Andale, S. Kaiser-Meyer-Olkin (KMO) Test for Sampling Adequacy. Available online: http://www.statisticshowto.com/ kaisermeyer-olkin/ (accessed on 24 December 2020).

16. Gorsuch, R.L. Using Bartlett's Significance Test to Determine the Number of Factors to Extract. Educ. Psychol. Meas. 1973, 33, 361-364. [CrossRef]

17. Makarewicz, A. Consumer behavior as a fundamental requirement for effective operations of companies. J. Int. Stud. 2013, 6, 103-109. [CrossRef] [PubMed]

18. Faller, H.; Kohlmann, T.; Zwingmann, C.; Maurischat, C. Exploratory and confirmatory factor analysis. Rehabilitation 2006, 45, 243-248. [CrossRef]

19. Mazzocchi, M. Factor Analysis and Principal Component Analysis. In Statistics for Marketing and Consumer Research; SAGE Publications Ltd.: London, UK, 2012. [CrossRef] 